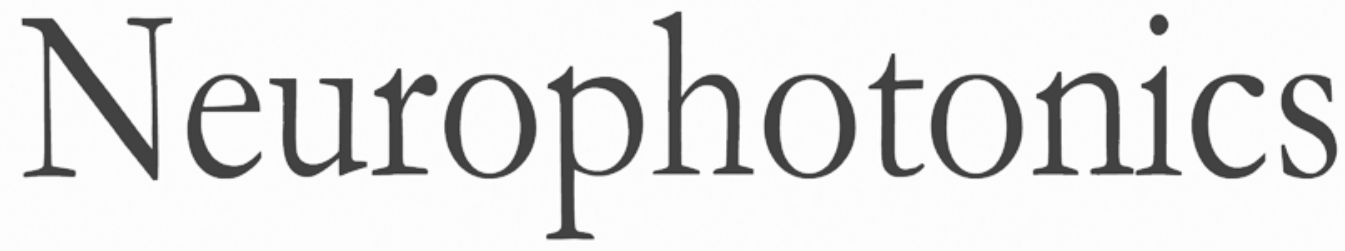

\title{
Mammalian cortical voltage imaging using genetically encoded voltage indicators: a review honoring professor Amiram Grinvald
}

\author{
Chenchen Song \\ Samuel Barnes \\ Thomas Knöpfel
}




\title{
Mammalian cortical voltage imaging using genetically encoded voltage indicators: a review honoring professor Amiram Grinvald
}

\author{
Chenchen Song, ${ }^{a}$ Samuel Barnes, ${ }^{a, b}$ and Thomas Knöpfel ${ }^{a, b, c, c}$ \\ Imperial College London, Laboratory for Neuronal Circuit Dynamics, London, United Kingdom \\ ${ }^{\mathrm{b}}$ Imperial College London, Division of Brain Sciences, London, United Kingdom \\ Institute of Biomedical Engineering, Imperial College London, Centre for Neurotechnology, South Kensington, London, United Kingdom
}

\begin{abstract}
The pioneering work of Amiram Grinvald established voltage-sensitive dye imaging (VSDI) in the mammalian cortex in the 1980s and inspired decades of cortical voltage imaging and the associated technological developments. The recent conception and development of genetically encoded voltage indicators (GEVIs) overcome many of the limitations of classical VSDI, and open experimental approaches that provide accruing support for orchestrated neuronal circuit dynamics of spatially distributed neuronal circuit underlying behaviors. We will review recent achievements using GEVIs to optically monitor the cortical activity in mammalian brains in vivo and provide a perspective for potential future directions. $\odot$ The Authors. Published by SPIE under a Creative Commons Attribution 3.0 Unported License. Distribution or reproduction of this work in whole or in part requires full attribution of the original publication, including its DOI. [DOI: 10.1117/1.NPh.4.3.031214]
\end{abstract}

Keywords: optical imaging; genetically encoded voltage indicators; voltage-sensitive fluorescent proteins; mesoscopic imaging; cortical dynamics.

Paper 17008SSVR received Jan. 17, 2017; accepted for publication Apr. 5, 2017; published online May 4, 2017.

\section{Introduction}

The brain, and in particular the human brain, is the most functionally complex mammalian organ. Fundamental to the function of the brain is short-lasting (in the range of milliseconds) changes of the plasma membrane potential of neurons. It has also long been recognized that approaches that enable the monitoring of these electrical activities are required to elucidate the brain's function in the processing and storage of information and the generation of behavior. Indeed, a multitude of available techniques were developed to achieve this goal, ranging from hightemporal resolution but with low-spatial coverage techniques, such as single-cell electrophysiology, to low-temporal resolution but with high-spatial coverage techniques, such as functional magnetic resonance imaging. Despite the functional values and clinical benefits of these techniques, their main limitation is the lack of combined temporal and spatial resolution and coverage. Understanding electrical activity at the single cell level is a fundamental goal of neuroscience, but large area monitoring of electrical activity at high-spatiotemporal resolution and coverage is also important for understanding the many emergent properties of neural network-level processing.

The early revolutionary work of Amiram Grinvald and colleagues took the important first step toward tackling the challenge of developing high-spatiotemporal resolution in the intact brain by establishing voltage-sensitive dye imaging (VSDI) of cortical electrical activities many decades ago. This approach relies on the topical application of voltage-sensitive dyes (VSDs) through a craniotomy to monitor the cortical activity. ${ }^{1}$ The more recently developed genetically encoded voltage

*Address all correspondence to: Thomas Knöpfel, E-mail: tknopfel@ knopfel-lab.net indicators (GEVIs) report, such as VSDs, membrane potential activity in the form of changes in optically detected fluorescence intensities while overcoming the methodological limitations associated with craniotomies and dye delivery.

GEVIs improve upon classic VSDs in several additional aspects: (i) they allow targeted expression in genetically identified cell classes with highly reproducible expression patterns across population, (ii) they provide reliable recordings from these specific cell populations over prolonged periods and across multiple sessions, and (iii) they permit noninvasive optical cortical monitoring in thin-cranium species such as mice. In addition, GEVIs provide direct real-time monitoring of neuronal electrical activity to uncover additional information including subthreshold activity and hyperpolarizing events, which cannot be detected using other genetically encoded optical indicators that report neuronal activity via surrogate signals (e.g., changes in calcium concentrations). As discussed in Antic et al., ${ }^{2}$ classic VSDs may still have advantages when applied in ex vivo preparation or in vivo for questions in which sub-millisecond time resolution is critical, for example, the investigation of plasticity involving changes in the timing of neurotransmission. ${ }^{3}$

In our lab we have generated a series of GEVIs termed voltage-sensitive fluorescent proteins (VSFPs) that are based on the voltage-sensor domain of Ciona intestinalis voltage-sensing phosphatase. $^{4-6}$ Based on this original design concept and several additional discoveries and contributions of others working in the field, there is now a growing palette of GEVIs. ${ }^{7}$ The molecular and biophysical properties of currently available GEVIs have been covered by several recent review articles. $^{2,6-8}$ In the context of the present review, we would like to highlight two technical considerations: (i) although many new GEVIs show promising properties when tested in cultured cells, only a few GEVIs have been rigorously validated 
in vivo. In our hands, the absolute amplitudes of optical GEVI signals are typically much smaller in vivo than in vitro (as is known for classical VSDs this is caused by a larger background signal), but typically comparable or better than those of widely used VSDs ${ }^{9}$ and (ii) many new and most-sensitive GEVIs are used with a single greenish fluorescence emission wavelength. As known for VSDs used before the development of "blue" [red and near infrared (NIR) fluorescence-emitting] VSDs, this feature causes complications in in vivo experiments due to the strong hemoglobin absorption in this wavelength range. ${ }^{10}$ GEVIs with two anticorrelated emission wavelengths [i.e., Förster resonance energy transfer (FRET)-based sensors] are, therefore, strongly preferable.

Here, we focus on the application of VSFPs in vivo in the mammalian system, with particular emphasis on transgenic animals expressing the VSFP Butterfly family ${ }^{11-13}$ of FRET-based GEVIs, to achieve targeted cortical monitoring of electrical activity of identified neuronal classes in head-fixed anesthetized and awake mice through noninvasive cranial window implants..$^{11,14}$

\section{Mesoscopic Monitoring of Cortical Activity}

Understanding how neuronal activity is transformed into complex behaviors, such as perception, sensory-motor integration, and eventual cognition and consciousness, is at the core of contemporary systems and circuit neuroscience. Cortical neuronal computation is distributed across large-scale networks over substantial cortical space, thus to optically observe circuit operations at the population level, i.e., mesoscopically, it is necessary to decipher information processing and to achieve functional mapping in the mammalian cortex.

The pioneering work of Grinvald and colleagues ${ }^{14}$ on optical monitoring of cortical activity using classic VSDs has inspired decades of mesoscopic voltage imaging to understand population-level neuronal computations, yet VSDI also has obvious methodological limitations. Cortical tissue comprises a heterogeneous neuronal population which VSDs label indiscriminately. Cortical VSDI is dominated by activity from the cortical glutamatergic populations because of their greater number, while contributing to diverse functions with no lesser importance are the GABAergic interneuron population which comprises only about $20 \%$ of the cortical cell population. This methodological challenge has been overcome by GEVI voltage imaging via genetic targeting of GEVIs to specific cell classes. This selective activity monitoring of specific populations is a crucial step toward an accurate description and reconstruction of dynamic cortical network interactions.

GEVI transgenic mice have achieved cell class-specific expression of the VSFP Butterflies, a ratiometric GEVI family that uses modulation of FRET between two fluorescent proteins as the voltage reporting mechanism. GEVI-based cortical voltage imaging is achieved through transcranial monitoring and can cover large cortical areas (often simultaneously across both hemispheres) in head-fixed anesthetized and awake animals [Fig. 1(a)]. The fluorescence intensities of the two fluorescent proteins are simultaneously monitored using two cameras after a dichroic mirror that splits emitted light into the donor and the acceptor spectral bands. Structural images show the prominent cortical vascular signatures that are used for preprocessing

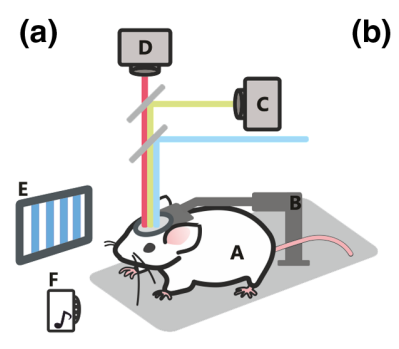

(c)

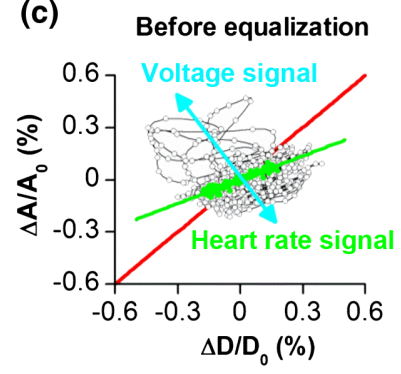

(b)
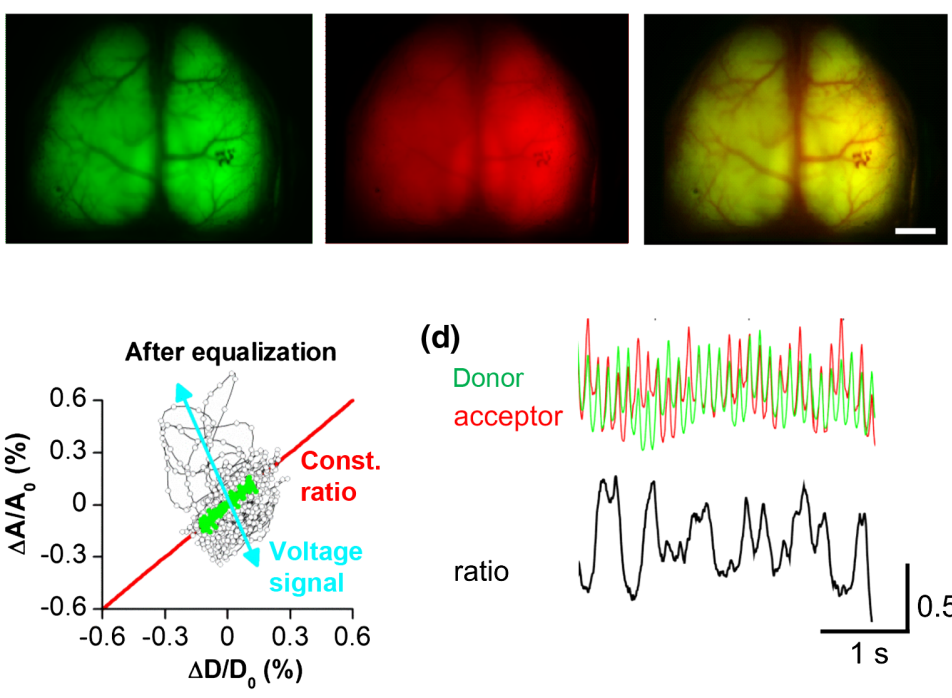

(d)

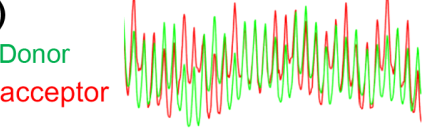

ratio

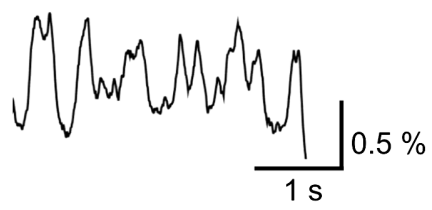

Fig. 1 Acquiring and preprocessing of epifluorescence signals from FRET-based ratiometric GEVIs. (a) A GEVI-expressing transgenic mouse $[A]$ is trained to be head-fixed $[B]$ after cranial window implant. Cortical activity is imaged under a dual-channel imaging set-up using two CCD cameras to monitor the fluorescence intensity changes of the FRET donor [C] and acceptor [D] signals simultaneously. Sensory stimulations (shown are visual gratings [E] and auditory tones [F] as examples) can be applied during optical imaging. (b) Left: mCitrine (donor) image; middle: mKate (acceptor) image; right: overlay of registered images. Scale bar $=3 \mathrm{~mm}$. (c) The signals derived from the two cameras reflect both voltage and hemodynamic responses. Heartbeat-related fluctuations in excitation and emission light absorption are observed in both donor and acceptor channel and are corrected by equalization of their amplitude before calculation of the acceptor/donor ratio. Left: before equalization; right: after equalization. (d) The FRET pair of fluorescent proteins anticorrelate in fluorescence intensity to reflect fluctuations in membrane potential during stimulus-free spontaneous activity to produce a ratiometric optical read-out. (b)(d) adapted with permission from Akemann et al. ${ }^{11}$ 
registration, and as landmarks for multisession registration and relocation of regions of interest [Fig. 1(b)].

Voltage indicator signals (both VSD and GEVIs) acquired with light at the visible range of wavelengths from the mammalian brain in vivo contain, in addition to the voltage signal, signals that are ascribed as hemodynamic response. The hemodynamic response has two principal components: (i) the pulsating blood flow causes rhythmic blood volume fluctuations at the heart beat frequency ${ }^{15}$ causing changes in absorbance in the optical path of excitation and emission light. Increase in blood volume results in decrease of indicator fluorescence and (ii) neuronal activity is associated with changes in hemoglobin oxygenation. The sign of this effect is wavelength dependent.

The dual emission feature of FRET-based GEVIs provides an opportunity not only to correct for hemodynamic signal components (separation of hemodynamic responses from the voltage signals) [Figs. 1(c) and 1(d)] but also to simultaneously monitor voltage and hemodynamic responses. ${ }^{11,14}$

\section{Cortex-Wide Intrinsic Population Activity}

A large body of work by Amiram Grinvald and colleagues has demonstrated that VSDI of mammalian cortex can resolve the dynamics of synchronized activities which occur in the absence of sensory input. ${ }^{16-20}$ This nonevoked activity is referred to as "spontaneous," "ongoing" or "internal activity," and is observed both in the developing and adult brains, in line with classical and more recent work using electrophysiological and calcium imaging techniques. ${ }^{21-24}$

Combined VSD optical imaging and electrophysiology have demonstrated that spontaneous synaptically-driven fluctuations of membrane potential of single neurons are often coherent time-locked events rather than independent processes,${ }^{25}$ indicating that such on-going cortical activity represents not just neuronal noise but reflects local and brain-wide orchestration of activities. ${ }^{16,26}$ Anatomically adjacent neurons can belong to different functional assemblies, yet the neuronal spontaneous firing is shown to be tightly linked to the cortical networks to which they belong. ${ }^{17}$ This is further supported by recent evidence suggesting a resemblance between spatial patterns of on-going activity motifs and activation maps obtained with sensory stimulation. $^{27}$

More recently, GEVI-based imaging confirmed many of the aforementioned observations. ${ }^{11,28,29}$ Cortex-wide mesoscopic voltage monitoring using the GEVI VSFP Butterfly 1.2 revealed spontaneous waves of activity of mouse layer two of three cortical pyramidal neuronal populations (Fig. 2). These waves traverse across the entire field of view, that is the dorsal aspect of the mouse cortex, and are observable across different anesthetic brain states, during slow wave sleep and during resting wakefulness. ${ }^{11,29-31}$ Interestingly, clustering analysis of the spatiotemporal patterns of these activity waves reveals a small set of recurrent motifs. Across different brain states, these motifs are conserved but with systematic changes in their dynamics. ${ }^{29}$

Experimental characterization of VSDI- and GEVI-based imaging of intrinsic activity patterns has been complemented by theoretical frameworks for neuronal circuit dynamics. ${ }^{30-32}$ The theory of criticality is one such potential framework that suggests that cortical networks self organize to operate at the dynamic boundary of local interactions and large-scale crosscortical synchronizations where the rules of interactions are scale invariant. ${ }^{33,34}$ Recent GEVI-based optical imaging of unperturbed cortex has shown that the features of spontaneous clusters of up-states (imaged as slow waves of population depolarizations) are in fact brain state dependent: criticality emerges with awakening, in which the dominance of long-range (cross cortical) interactions characteristic for slow wave sleep and anesthesia decreases $^{30}$ (Fig. 3). Analysis of GEVI optical imaging data in the framework of information theory supported the postulation that information capacity within the cortical network increases with the emergence of criticality. ${ }^{31,35}$

\section{Cortical Representation of Sensory Information}

The physiological mechanisms underlying cortical sensory processing have long been established from using single cell- (a)

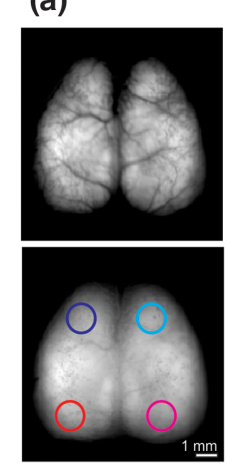

(b)

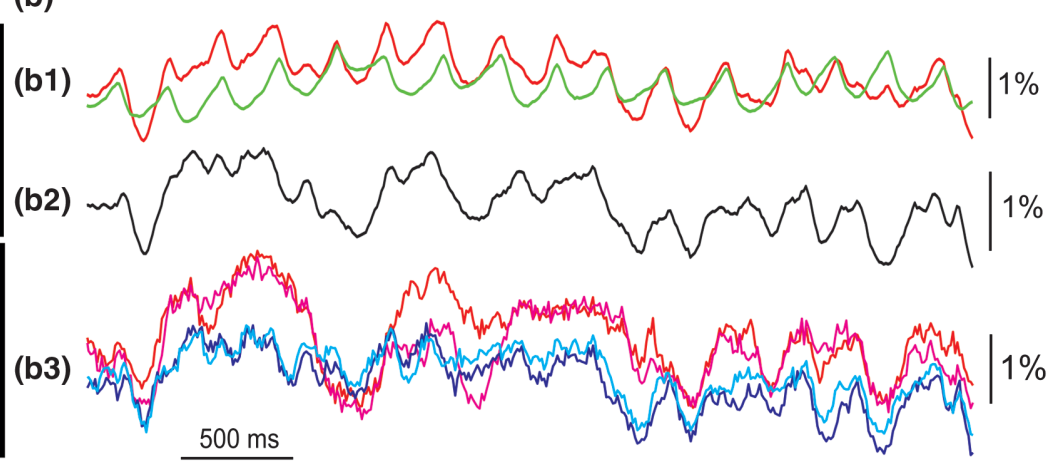

Fig. 2 Intrinsic activity in the absence of sensory stimuli monitored in transgenic mice expressing VSFP Butterfly 1.2 in layer II/III pyramidal neurons. (a) Dorsal view over both cortical hemispheres of a mouse with a chronically implanted transcranial window, captured through the FRET donor (upper) and acceptor (lower) channels. Four ROls are outlined as: (i) left motor cortex (navy), (ii) right motor cortex (cyan), (iii) left visual cortex (red), and (iv) right visual cortex (pink). (b) Intrinsic activity imaged across both hemispheres in the absence of sensory stimulation under light sedation. Upper: individual donor (green) and acceptor (red) fluorescence signals averaged across both hemispheres; middle: ratiometric voltage signal across both hemispheres; lower: ratiometric voltage signal of intrinsic activity across the four ROls [left motor cortex (navy), right motor cortex (cyan), left visual cortex (red), and right visual cortex (pink)] as outlined in A lower, showing isotopic cortical activity traversing across large distance in both hemispheres. These data were collected as described in Akemann et al. ${ }^{11}$ and Carandini et al. ${ }^{14}$ 
(a)

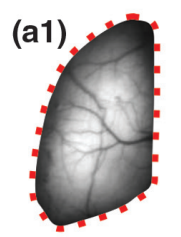

(a2)
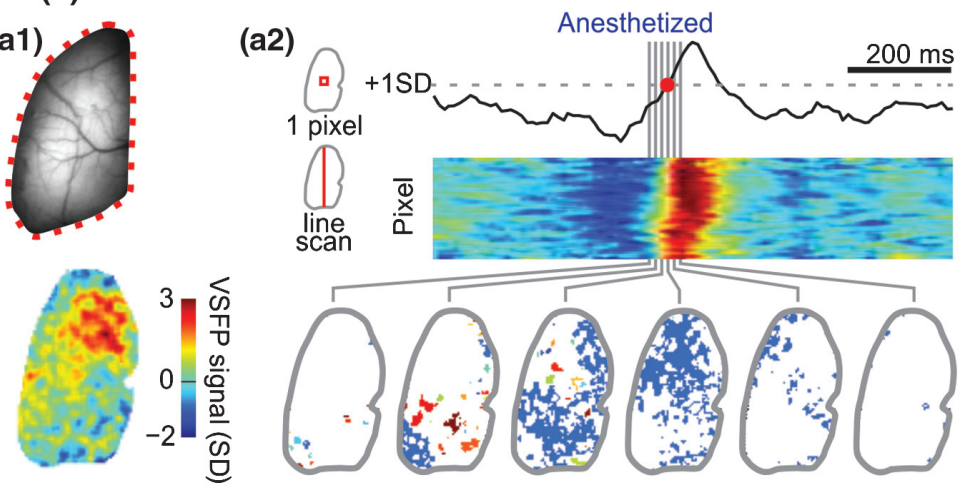

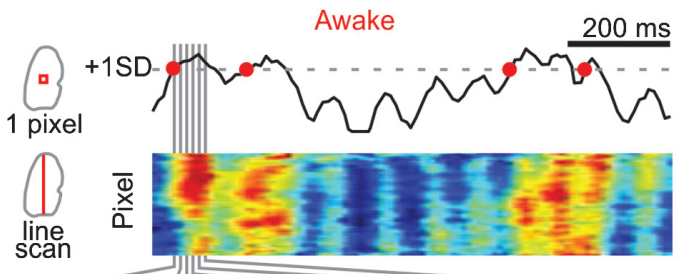

(b)

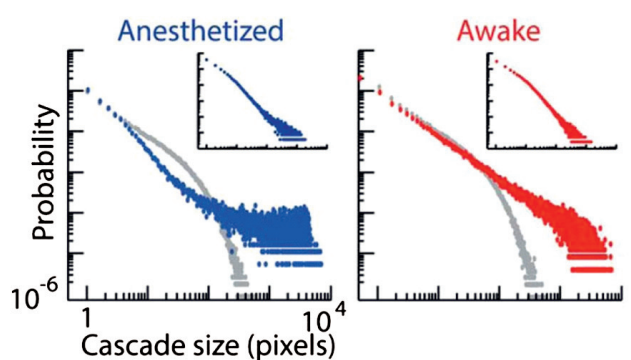

(c)
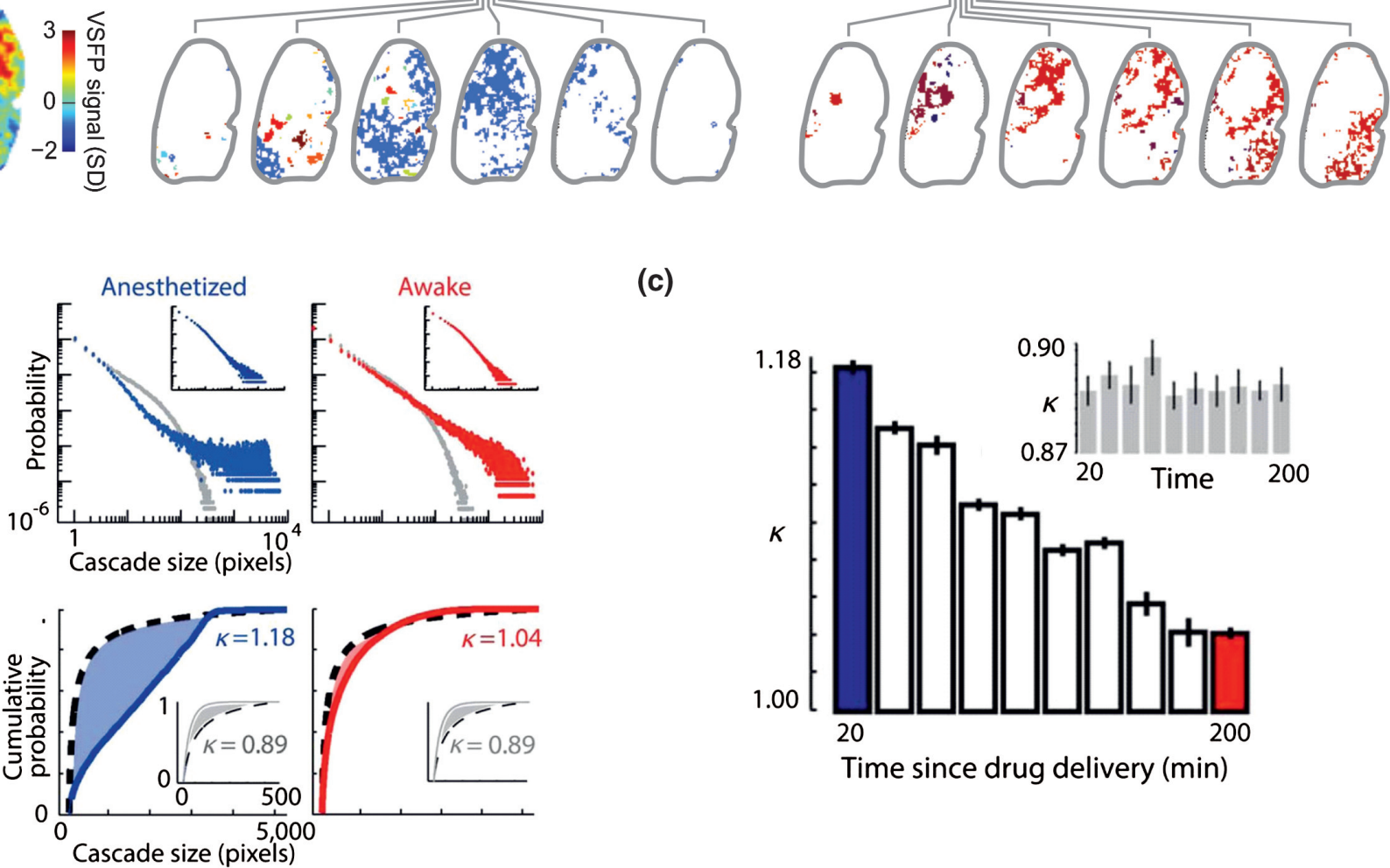

Fig. 3 Spontaneously occurring cascades of population depolarizations show brain state-dependent emergence of criticality. (a) Voltage imaging in a head-fixed mouse through a dorsal transcranial window (one hemisphere shown). Upper: baseline fluorescence image. Lower: map of VSFP Butterfly 1.2 voltage signal for a 20-ms snapshot. (a1) Data from an anesthetized mouse (left) and a mouse in resting wakefulness state (right). Upper: voltage imaging traces from one pixel $(33 \times 33 \mu \mathrm{m}$ cortical area). A pixel is considered to be active at times when the signal crosses a threshold from below (red circles). Middle: voltage signals from a vertical line of pixels reveal spatiotemporally contiguous activity; color code as in a1. Lower: consecutive snapshots of cascades of active pixels during a 120-ms period. Each cascade has been labeled with a unique color. (b) Cascade size probability distributions approach power-law form during the recovery from anesthesia. Upper: probability distribution of cascade sizes, in number of active pixels, in anesthetized (left, blue), and awake resting (right, red) states. Each data point represents the probability $\operatorname{Pr}(z)$ of observing a cascade of size $z$. Very large cascades were prevalent in the anesthetized state. The probability distribution for resting awake-state mouse is close to a power law. Gray points show probability distributions after randomized circular permutation of single pixel traces relative to each other, a control with no cross-pixel correlations. Data presented are for the 10 image sequences recorded over a 20 -min period in anesthetized states (20 to $40 \mathrm{~min}$ ) and in resting states (200 to $220 \mathrm{~min}$ ) in one mouse. Insets show cluster size distributions for the anesthetized and resting states. Lower: cumulative probability distributions for anesthetized (left, blue) and resting (right, red) states. Deviations from a reference power law with exponent -1.5 (black dashed lines) are quantified by $\kappa$, which measures the gap (shaded area) between the measured distribution and the reference distribution. The closer $\kappa$ is to 1 , the closer the distribution is to the reference. Shuffled control distributions for both states showed similar deviation from the reference distribution, with $\kappa<1$ (insets, gray). (c) Values of $\kappa$ calculated for image sequences recorded over 20-min periods, and shuffled data (inset, gray). Error bars represent SEM of 10 consecutive sequences. Adapted with permission from Scott et al. . $^{30}$

level electrophysiology, ${ }^{36-39}$ but how sensory stimuli are processed involving top-down influences and long-range connections required monitoring representations across larger cortical areas. Cortical VSDI, as inspired by the early work of Grinvald and colleagues, ${ }^{40}$ has contributed much to our understanding of sensory neurophysiology at the level of local cortical networks but also, perhaps even more importantly, at the level of cortex-wide interarea connectivities. With the aforementioned stochastic ongoing spontaneous activity averaged out over repeated presentation of the sensory stimulus, 
VSDI has generated maps of sensory representation in the visual, ${ }^{41-45}$ auditory, ${ }^{46-48}$ somatosensory cortices, ${ }^{15,49-51}$ and the olfactory bulb, ${ }^{52}$ and provided basic insights as to the cortical processing of neuronal information.

Following its initial application in invertebrates, amphibians, cats, and monkeys, the use of VSDI in cortical sensory processing was extended to rodents, in which earlier work using VSDI had established that peripheral stimulations of whiskers elicit a "field of activity" extending over in the somatosensory cortical columns. ${ }^{50}$ Subsequent work confirmed this observation, and at an improved spatiotemporal resolution, resolved the initial activity to be confined to a single column. ${ }^{49}$ The physiological extent of this activity spread, as mapped by VSDI, is correlated to the stimulation strength, weaker whisker deflections elicit depolarization confined to a single barrel column, intermediate deflections cause depolarization to spread into adjacent barrel columns preferentially asymmetrically along whisker rows, whereas large deflections generate a depolarizing event spreading over the barrel field. Later work also confirmed the triphasic response of this elicited activity observed by Kleinfeld and Delaney, ${ }^{50}$ a rapid initial depolarization phase, followed by hyperpolarization, and ending with a long-lasting rebound depolarization. ${ }^{51,53}$ The stimulus-sensitive hyperpolarizing phase appeared in the form of an asymmetrical ring that is more distant from the epicentre, providing spatiotemporal insights into the mechanism by which the cortex processes sensory information, such as lateral inhibition $^{54,55}$ and gain normalization. ${ }^{56-58}$

Sensory perception at the cortex-wide (interarea) level relies on the dynamic function of long-range connections, ${ }^{59-61}$ and VSDI contributed much to connectivity mapping and the characterization of cortical activity propagation in vivo. ${ }^{62-64}$ Longrange cortical interactions have been shown using VSDI in experiments in which brief whisker deflection elicits an initial depolarizing response in the contralateral primary somatosensory (S1) barrel cortex that generates secondary localized activity in "satellite regions," Secondary activity is also observed in the ipsilateral cortical regions, resulting in eventual apparent bilaterally symmetrical depolarizing activity spreading to other cortical regions with complex spatiotemporal dynamics. ${ }^{65}$

Connections that are involved in higher-order sensory processing extend beyond the supragranular layers into deeper cortical layers and subcortical structures (e.g., thalamus). Longdistance functional connectomics have been facilitated by combining VSDI of superficial cortical layers with photostimulation of deeper cortical layers using optogenetic actuators expressed in restricted cell classes. ${ }^{66,67}$ This approach capitalizes on the superior target selectivity of optogenetic stimulation compared with electrical microstimulation, but does not resolve the heterogeneous nature of VSDI signals and the restricted monitoring to superficial cortical layers, in which all cell types of the superficial layers and the apical dendrites of the deeper layers are indiscriminately represented in the optical voltage signal.

GEVI-based voltage imaging therefore serves as a perfect opportunity for monitoring neuronal responses in both a cell class-specific (e.g., pyramidal neurons versus GABAergic interneurons) and anatomically defined (e.g., dendrites of supragranular layer pyramids versus apical dendrites from supgranular pyramidal cells) manner, to achieve precise reconstruction of the functional dynamics of sensory processing. Functional mapping of sensory processing in layer II/III pyramidal populations

using ratiometric GEVI have been realized initially using VSFP2, ${ }^{28}$ and subsequently using VSFP Butterfly 1.2.11,14 This was later also achieved via transgenic strategies ${ }^{12,13}$ for enhanced stringent control over the optical signal origin. In transgenic mice expressing VSFP Butterfly 1.2 in layer II/III pyramidal neurons, the binocular presentation of horizontal drifting gratings elicited isotopic depolarizing activity initiating from the primary visual cortices in both hemispheres [Fig. 4(a)] which is temporally comparable with previous observations using electrophysiological measures. ${ }^{68}$

Somatosensory stimulation of head-fixed transgenic animals expressing the GEVI chimeric VSFP Butterfly in all cortical pyramidal neurons have also produced rapid depolarizing homeotopic response in the somatosensory cortex [Fig. 4(b)]. This depolarizing response is observed both in the contralateral and the ipsilateral barrel cortices with the ipsilateral responses appearing after a small delay, similar to previous observations using VSDI. ${ }^{65}$ Expression of the same GEVI in all cortical GABAergic cell types allowed the selective monitoring of somatosensory processing in the interneuron populations only, providing insights into the distinctive excitatory and inhibitory components of dynamic neuronal circuits. Because the balance between excitation and inhibition in the neuronal circuits have major implications for sensory processing, ${ }^{69-71}$ this ability to discern the excitatory and inhibitory components of sensory

(a)

(a1)
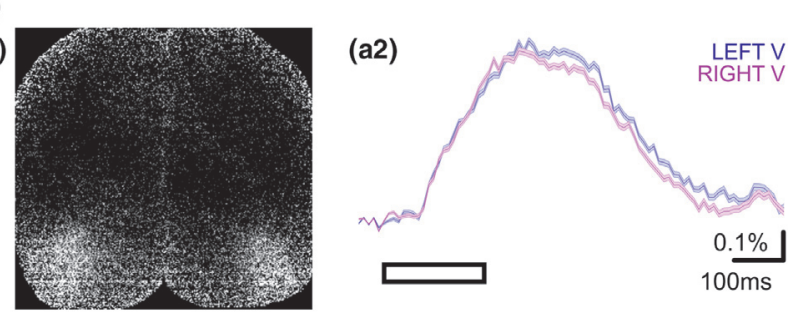

(b)
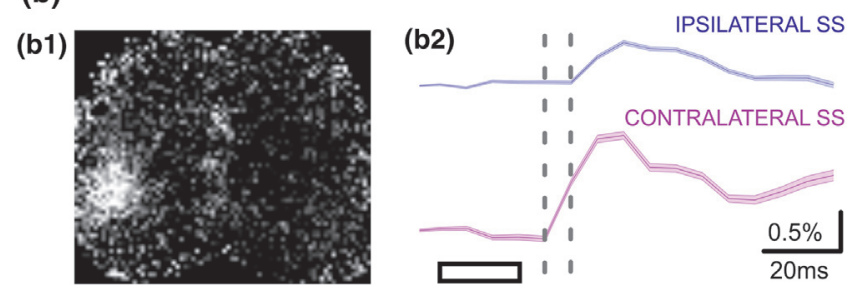

Fig. 4 Cortical representation of sensory stimuli in slightly sedated mice. (a) Cortical responses to bilateral visual stimulation (200-ms horizontal moving grating) in a transgenic mouse expressing VSFP Butterfly 1.2 in layer II/III pyramidal population. (a1): $\Delta R / R$ snapshot showing peak depolarizing response in both visual cortices following binocular sensory stimulation. (a2): Ratiometric depolarizing responses from the left and right visual cortex ROls, averaged across 40 trials $( \pm$ SEM). (b) Cortical response to right whisker stimulation (20 ms air puff to whole trimmed whisker field) in a transgenic mouse expressing chimeric VSFP Butterfly in pyramidal neurons across all cortical layers. (b1): $\Delta R / R$ snapshot showing peak depolarizing response in the contralateral somatosensory cortex following sensory stimulation. (b2): Ratiometric depolarizing response from the contralateral and ipsilateral somatosensory ROls, averaged across 50 trials $( \pm$ SEM). Fast depolarizing response is initially observed in the contralateral ROI, and subsequently in the ipsilateral ROI with a minor delay. Gray dotted lines demark time period represented by the snapshot shown in b1. Note that the ipsilateral response appears only in the subsequent frame. Note that the different response timescales in (a) and (b). Mice are sedated. These data were collected as described in Akemann et al. ${ }^{11}$ and Carandini et al. ${ }^{14}$ 
processing also has profound implications for understanding normal neurophysiology ${ }^{72,73}$ as well as diseased states. ${ }^{74}$

\section{2-Photon GEVI Voltage Imaging}

Voltage maps obtained with classical VSDs in wide-field mode show, at best, blurred cellular morphologies due to out-of-focus light and light scattering. The optically reported membrane voltage in vivo in wide-field mode imaging experiments is a compound voltage reflecting contributions of stained membranes within a larger tissue volume delimited axially by the penetration length of visible light $(<200 \mu \mathrm{m})$. Although a visualization of coarse compound activity often provides a useful representation of cortical dynamics by emphasizing the major modes of activity shared by a larger set of neurons, there are other questions requiring sharper resolution.

The foremost optical approach to improve spatial resolution and deliver unblurred optical sections of micrometer thickness up to larger tissue depth is 2-photon microscopy. ${ }^{75}$ However, 2-photon voltage imaging has been lagging as a monitoring method, mostly because of the low photon counts expected in the 2-photon image scan acquisition mode at fast frame rate unavoidably leading to high-photon shot noise. ${ }^{76}$ Nevertheless, in vitro studies recently demonstrated single-trial sensitivity for recording of action potentials in axonal terminal arbors, ${ }^{77}$

(a)
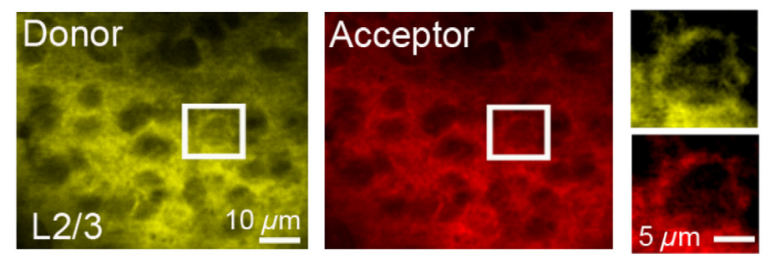

(b)
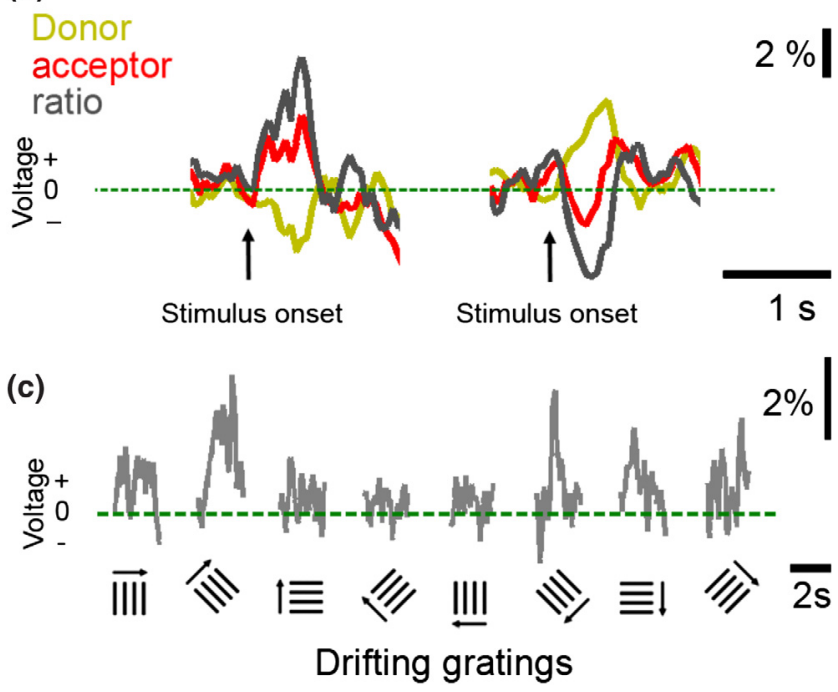

Fig. 5 2-Photon in vivo voltage imaging of sensory processing under sedation. (a) Example of in vivo cortical regions from a transgenic mouse expressing chimeric VSFP Butterfly in all pyramidal neurons. A single layer II/III pyramidal neuron is outlined in the ROI. (b) Singletrial in vivo voltage responses to auditory stimuli from the pyramidal neuron in the identified ROI in (a) (right), where different auditory stimuli can elicit either depolarizing response (left) or hyperpolarizing response (right). (c) Example of average voltage responses to visual stimulation (drifting gratings of different orientations; number of trials $=10$ ). These data are unpublished examples generated using the methods described in Akemann et al. ${ }^{81}$ back-propagated action potentials in single spines, ${ }^{78}$ and spontaneous and evoked somatic potentials in neurons in acute brain slice $^{79}$ using 2-photon imaging of VSD variants. On the other hand, available 2-photon imaging data of sensory-evoked activity in somatosensory and visual cortex remained poor in signalto-noise requiring extensive trial averages $(>100)$ to surpass the noise barrier. ${ }^{76,80}$ More recently, 2-photon voltage imaging using GEVIs was explored with promising conclusions. ${ }^{80-82}$

We established the feasibility of 2-photon voltage imaging in the mouse cortex at single cell resolution using Butterfly GEVIs $^{81}$ (Fig. 5). The optical voltage signals in these recordings have a dynamic range of $1 \%$ to $3 \%$, about two to three times larger than in 1-photon recording (but 1 to 2 orders of magnitude smaller than typical calcium imaging signals). The increased signals observed with 2-photon imaging compared with 1-photon excitation modes can be explained by less nonresponsive background fluorescence in the 2-photon case. The dynamic range of 2-photon voltage signals may be small compared with those of calcium indicators but in addition to the modest signal size, cellular level voltage imaging is further complicated by the fact that most of the volume averaged optical voltage signals is generated by neuronal dendrites..$^{15,49,83}$ This latter aspect could be resolved by the promising efforts in attaining optimum sparse indicator expression in individual neurons in vivo.

\section{Future Directions}

It has been pointed out that the first GEVI was published around the same time as the first genetically encoded calcium indicator (GECI), but it may be interesting to note that the first GEVIs did not function in mammalian cells, whereas the first GECIs did. Iterative improvement of GECIs resulted in more probes with greater sensitivity that are now widely used to monitor calcium transients in the live mammalian brain. ${ }^{84}$ It is reasonable to expect that the development of GEVIs will follow this path. Indeed, there are intense current efforts by several groups and consortia toward improved GEVIs and associated technologies, many of which are supported via the BRAIN initiative. But voltage imaging will remain more challenging and more difficult than the widely and successfully used calcium imaging approaches, be it only because the signals of interest are typically faster (voltage transients versus calcium transients). Beyond efforts to improve the effective sensitivity of GEVIs, current efforts focus on sparse targeting (to facilitate allocation of signals to individual neurons), subcellular targeting selectivity (soma dendritic versus axonal membranes to maximize the ratio between signal photons and noise photons) and indicator color variants that extend into the NIR. NIR GEVIs would be tremendously useful for combination with blue/greenish lightmediated optogenetic photostimulation and deep tissue imaging toward further delineating the functional causal mechanisms underlying in vivo neurophysiology.

\section{Disclosures}

The authors declare neither relevant financial interest nor other potential conflicts of interest.

\section{Acknowledgments}

We would like to thank all current members of the Knöpfel Lab for discussions, and the funding bodies that support the work in our lab (HFSP RGP0052/2012, NIH U01MH109091, and NIH 1U01NS099573) for their financial support. SJB was funded by the Edmond J. Safra Foundation. 


\section{References}

1. A. Grinvald and R. Hildesheim, "VSDI: a new era in functional imaging of cortical dynamics," Nat. Rev. Neurosci. 5, 874-885 (2004).

2. S. D. Antic, R. M. Empson, and T. Knopfel, "Voltage imaging to understand connections and functions of neuronal circuits," J. Neurophysiol. 116, 135-152 (2016).

3. S. J. Barnes et al., "Delayed and temporally imprecise neurotransmission in reorganizing cortical microcircuits," J. Neurosci. 35, 9024-9037 (2015).

4. D. Dimitrov et al., "Engineering and characterization of an enhanced fluorescent protein voltage sensor," PLoS One 2, e440 (2007).

5. B. J. Baker et al., "Genetically encoded fluorescent sensors of membrane potential," Brain Cell Biol. 36, 53-67 (2008).

6. T. Knöpfel, Y. Gallero-Salas, and C. Song, "Genetically encoded voltage indicators for large scale cortical imaging come of age," Curr. Opin. Chem. Biol. 27, 75-83 (2015).

7. W. Akemann et al., "Route to genetically targeted optical electrophysiology: development and applications of voltage-sensitive fluorescent proteins," Neurophotonics 2, 021008 (2015).

8. M. Z. Lin and M. J. Schnitzer, "Genetically encoded indicators of neuronal activity," Nat. Neurosci. 19, 1142-1153 (2016).

9. H. Mutoh et al., "Comparative performance of a genetically-encoded voltage indicator and a blue voltage sensitive dye for large scale cortical voltage imaging," Front Cell Neurosci. 9, 147 (2015).

10. E. M. Hillman, "Optical brain imaging in vivo: techniques and applications from animal to man," J. Biomed. Opt. 12, 051402 (2007).

11. W. Akemann et al., "Imaging neural circuit dynamics with a voltagesensitive fluorescent protein," J. Neurophysiol. 108, 2323-2337 (2012).

12. L. Madisen et al., "Transgenic mice for intersectional targeting of neural sensors and effectors with high specificity and performance," Neuron 85, 942-958 (2015).

13. Y. Mishina et al., "Exploration of genetically encoded voltage indicators based on a chimeric voltage sensing domain," Front. Mol. Neurosci. 7, 78 (2014).

14. M. Carandini et al., "Imaging the awake visual cortex with a genetically encoded voltage indicator," J. Neurosci. 35, 53-63 (2015).

15. H. S. Orbach, L. B. Cohen, and A. Grinvald, "Optical mapping of electrical activity in rat somatosensory and visual cortex," J. Neurosci. 5, 1886-1895 (1985).

16. A. Arieli et al., "Dynamics of ongoing activity: explanation of the large variability in evoked cortical responses," Science 273, 1868-1871 (1996).

17. M. Tsodyks et al., "Linking spontaneous activity of single cortical neurons and the underlying functional architecture," Science 286, 1943-1946 (1999).

18. T. Kenet et al., "Spontaneously emerging cortical representations of visual attributes," Nature 425, 954-956 (2003).

19. T. Deneux and A. Grinvald, "Milliseconds of sensory input abruptly modulate the dynamics of cortical states for seconds," Cereb. Cortex 27, 1-15 (2016).

20. C. C. Petersen et al., "Interaction of sensory responses with spontaneous depolarization in layer $2 / 3$ barrel cortex," Proc. Natl. Acad. Sci. U. S. A. 100, 13638-13643 (2003).

21. M. Steriade, "Central core modulation of spontaneous oscillations and sensory transmission in thalamocortical systems," Curr. Opin. Neurobiol. 3, 619-625 (1993).

22. A. Destexhe, D. Contreras, and M. Steriade, "Spatiotemporal analysis of local field potentials and unit discharges in cat cerebral cortex during natural wake and sleep states," J. Neurosci. 19, 4595-4608 (1999).

23. I. Lampl, I. Reichova, and D. Ferster, "Synchronous membrane potential fluctuations in neurons of the cat visual cortex," Neuron 22, 361-374 (1999)

24. S. J. Barnes et al., "Subnetwork-specific homeostatic plasticity in mouse visual cortex in vivo," Neuron 86, 1290-1303 (2015).

25. A. Arieli et al., "Coherent spatiotemporal patterns of ongoing activity revealed by real-time optical imaging coupled with single-unit recording in the cat visual cortex," J. Neurophysiol. 73, 2072-2093 (1995).

26. A. Grinvald et al., "Neuronal assemblies: single cortical neurons are obedient members of a huge orchestra," Biopolymers 68, 422-436 (2003).
27. M. H. Mohajerani et al., "Spontaneous cortical activity alternates between motifs defined by regional axonal projections," Nat. Neurosci. 16, 1426-1435 (2013).

28. W. Akemann et al., "Imaging brain electric signals with genetically targeted voltage-sensitive fluorescent proteins," Nat. Methods 7, 643-649 (2010).

29. D. Shimaoka, C. Song, and T. Knöpfel, "State-dependent modulation of slow wave motifs towards awakening," Front. Cell. Neurosci. 11, 108 (2017).

30. G. Scott et al., "Voltage imaging of waking mouse cortex reveals emergence of critical neuronal dynamics," J. Neurosci. 34, 16611-16620 (2014).

31. E. D. Fagerholm et al., "Cortical entropy, mutual information and scalefree dynamics in waking mice," Cereb. Cortex 26, 3945-3952 (2016).

32. Y. F. Sit et al., "Complex dynamics of V1 population responses explained by a simple gain-control model," Neuron 64, 943-956 (2009).

33. J. P. Sethna, K. A. Dahmen, and C. R. Myers, "Crackling noise," Nature 410, 242-250 (2001).

34. W. L. Shew and D. Plenz, "The functional benefits of criticality in the cortex," Neuroscientist 19, 88-100 (2013).

35. W. L. Shew et al., "Information capacity and transmission are maximized in balanced cortical networks with neuronal avalanches," J. Neurosci. 31, 55-63 (2011).

36. V. B. Mountcastle, "Modality and topographic properties of single neurons of cat's somatic sensory cortex," J. Neurophysiol. 20, 408-434 (1957).

37. V. B. Mountcastle, P. W. Davies, and A. L. Berman, "Response properties of neurons of cat's somatic sensory cortex to peripheral stimuli," J. Neurophysiol. 20, 374-407 (1957).

38. D. H. Hubel and T. N. Wiesel, "Receptive fields, binocular interaction and functional architecture in the cat's visual cortex," J. Physiol. 160, 106-154 (1962).

39. D. H. Hubel and T. N. Wiesel, "Ferrier lecture. Functional architecture of macaque monkey visual cortex," Proc. R. Soc. London B Biol. Sci. 198, 1-59 (1977).

40. A. Grinvald et al., "Functional architecture of cortex revealed by optical imaging of intrinsic signals," Nature 324, 361-364 (1986).

41. M. T. Lippert et al., "Methods for voltage-sensitive dye imaging of rat cortical activity with high signal-to-noise ratio," J. Neurophysiol. 98, 502-512 (2007)

42. P. E. Roland et al., "Cortical feedback depolarization waves: a mechanism of top-down influence on early visual areas," Proc. Natl. Acad. Sci. U. S. A. 103, 12586-12591 (2006).

43. D. Shoham et al., "Imaging cortical dynamics at high spatial and temporal resolution with novel blue voltage-sensitive dyes," Neuron 24, 791-802 (1999).

44. D. Sharon and A. Grinvald, "Dynamics and constancy in cortical spatiotemporal patterns of orientation processing," Science 295, 512-515 (2002).

45. A. Grinvald et al., "Cortical point-spread function and long-range lateral interactions revealed by real-time optical imaging of macaque monkey primary visual cortex," J. Neurosci. 14, 2545-2568 (1994).

46. H. Sawatari et al., "Identification and characterization of an insular auditory field in mice," Eur. J. Neurosci. 34, 1944-1952 (2011).

47. W. J. Song et al., "Cortical intrinsic circuits can support activity propagation through an isofrequency strip of the guinea pig primary auditory cortex," Cereb. Cortex 16, 718-729 (2006).

48. M. Nishimura et al., "New field with tonotopic organization in guinea pig auditory cortex," J. Neurophysiol. 97, 927-932 (2007).

49. C. C. Petersen, A. Grinvald, and B. Sakmann, "Spatiotemporal dynamics of sensory responses in layer $2 / 3$ of rat barrel cortex measured in vivo by voltage-sensitive dye imaging combined with whole-cell voltage recordings and neuron reconstructions," J. Neurosci. 23, 1298-1309 (2003).

50. D. Kleinfeld and K. R. Delaney, "Distributed representation of vibrissa movement in the upper layers of somatosensory cortex revealed with voltage-sensitive dyes," J. Comp. Neurol. 375, 89-108 (1996).

51. D. Derdikman et al., "Imaging spatiotemporal dynamics of surround inhibition in the barrels somatosensory cortex," J. Neurosci. 23, 3100-3105 (2003).

52. H. Spors and A. Grinvald, "Spatio-temporal dynamics of odor representations in the mammalian olfactory bulb," Neuron 34, 301-315 (2002). 
53. I. Takashima, R. Kajiwara, and T. Iijima, "Voltage-sensitive dye versus intrinsic signal optical imaging: comparison of optically determined functional maps from rat barrel cortex," Neuroreport 12, 2889-2894 (2001).

54. M. Wehr and A. M. Zador, "Balanced inhibition underlies tuning and sharpens spike timing in auditory cortex," Nature 426, 442-446 (2003).

55. C. Blakemore and E. A. Tobin, "Lateral inhibition between orientation detectors in the cat's visual cortex," Exp. Brain Res. 15, 439-440 (1972).

56. D. J. Heeger, "Normalization of cell responses in cat striate cortex," Visual Neurosci. 9, 181-197 (1992).

57. T. K. Sato et al., "An excitatory basis for divisive normalization in visual cortex," Nat. Neurosci. 19, 568-570 (2016).

58. T. K. Sato, M. Hausser, and M. Carandini, "Distal connectivity causes summation and division across mouse visual cortex," Nat. Neurosci. 17, 30-32 (2014).

59. T. Mao et al., "Long-range neuronal circuits underlying the interaction between sensory and motor cortex," Neuron 72, 111-123 (2011).

60. B. M. Hooks et al., "Organization of cortical and thalamic input to pyramidal neurons in mouse motor cortex," J. Neurosci. 33, 748-760 (2013).

61. L. Petreanu et al., "Channelrhodopsin-2-assisted circuit mapping of long-range callosal projections," Nat. Neurosci. 10, 663-668 (2007).

62. R. Aronoff et al., "Long-range connectivity of mouse primary somatosensory barrel cortex," Eur. J. Neurosci. 31, 2221-2233 (2010).

63. M. H. Mohajerani et al., "Mirrored bilateral slow-wave cortical activity within local circuits revealed by fast bihemispheric voltage-sensitive dye imaging in anesthetized and awake mice," J. Neurosci. 30, 3745-3751 (2010).

64. D. J. Wallace and B. Sakmann, "Plasticity of representational maps in somatosensory cortex observed by in vivo voltage-sensitive dye imaging," Cereb. Cortex 18, 1361-1373 (2008).

65. I. Ferezou et al., "Spatiotemporal dynamics of cortical sensorimotor integration in behaving mice," Neuron 56, 907-923 (2007).

66. S. Q. Lo et al., "All-optical mapping of barrel cortex circuits based on simultaneous voltage-sensitive dye imaging and channelrhodopsinmediated photostimulation," Neurophotonics 2, 021013 (2015).

67. D. H. Lim et al., "In vivo large-scale cortical mapping using channelrhodopsin-2 stimulation in transgenic mice reveals asymmetric and reciprocal relationships between cortical areas," Front. Neural Circuits 6, 11 (2012).

68. C. M. Niell and M. P. Stryker, "Highly selective receptive fields in mouse visual cortex," J. Neurosci. 28, 7520-7536 (2008).

69. J. E. Heiss et al., "Shift in the balance between excitation and inhibition during sensory adaptation of S1 neurons," J. Neurosci. 28, 1332013330 (2008).

70. W. B. Wilent and D. Contreras, "Dynamics of excitation and inhibition underlying stimulus selectivity in rat somatosensory cortex," Nat. Neurosci. 8, 1364-1370 (2005).

71. G. K. Wu et al., "Lateral sharpening of cortical frequency tuning by approximately balanced inhibition," Neuron 58, 132-143 (2008).

72. J. S. Isaacson and M. Scanziani, "How inhibition shapes cortical activity," Neuron 72, 231-243 (2011).
73. B. Haider et al., "Neocortical network activity in vivo is generated through a dynamic balance of excitation and inhibition," J. Neurosci. 26, 4535-4545 (2006).

74. O. Yizhar et al., "Neocortical excitation/inhibition balance in information processing and social dysfunction," Nature 477, 171-178 (2011).

75. W. Denk, J. H. Strickler, and W. W. Webb, "Two-photon laser scanning fluorescence microscopy," Science 248, 73-76 (1990).

76. B. Kuhn, W. Denk, and R. M. Bruno, "In vivo two-photon voltage-sensitive dye imaging reveals top-down control of cortical layers 1 and 2 during wakefulness," Proc. Natl. Acad. Sci. U. S. A. 105, 7588-7593 (2008).

77. J. A. Fisher et al., "Two-photon excitation of potentiometric probes enables optical recording of action potentials from mammalian nerve terminals in situ," J. Neurophysiol. 99, 1545-1553 (2008).

78. C. D. Acker, P. Yan, and L. M. Loew, "Single-voxel recording of voltage transients in dendritic spines," Biophys. J. 101, L11-L13 (2011).

79. P. Yan et al., "Palette of fluorinated voltage-sensitive hemicyanine dyes," Proc. Natl. Acad. Sci. U. S. A. 109, 20443-20448 (2012).

80. K. F. Ahrens et al., "Two-photon scanning microscopy of in vivo sensory responses of cortical neurons genetically encoded with a fluorescent voltage sensor in rat," Front. Neural Circuits 6, 15 (2012).

81. W. Akemann et al., "Two-photon voltage imaging using a genetically encoded voltage indicator," Sci. Rep. 3, 2231 (2013).

82. H. H. Yang et al., "Subcellular imaging of voltage and calcium signals reveals neural processing in vivo," Cell 166, 245-257 (2016).

83. A. Grinvald, A. Manker, and M. Segal, "Visualization of the spread of electrical activity in rat hippocampal slices by voltage-sensitive optical probes," J. Physiol. 333, 269-291 (1982).

84. T. W. Chen et al., "Ultrasensitive fluorescent proteins for imaging neuronal activity," Nature 499, 295-300 (2013).

Chenchen Song studied medical biochemistry and cognitive neuroscience at the University of Birmingham, UK. She then joined the Knöpfel Lab at Imperial College London, UK, where she works on monitoring cortical activity during behavior using optogenetic imaging approaches.

Samuel Barnes graduated from Oxford University in 2006 before completing his PhD at the MRC Centre for Neurodegeneration King's College London. For his postdoctoral work he investigated adult homeostatic plasticity with Dr. Tara Keck at the University College London. In 2015, he took up the Edmond Safra Scholarship position at Imperial College London and works in close collaboration with professor Knöpfel's group to investigate multisensory integration in neural microcircuits.

Thomas Knöpfel received his MD degree from the University of UIm, Germany, and his PhD in brain science from the University of Zürich, Switzerland. He also graduated in physics. After promotion to associate professor of physiology at the University of Zürich, he worked for 3 years for a Swiss Pharmaceutical Company leading a drug discovery project in the field of glutamate receptors. From 1998 to 2013, he worked at the RIKEN Brain Science Institute, Japan, to develop optogenetic monitoring tools for the analysis of neuronal circuit mechanisms. Since 2013, he has been a chair of optogenetics and circuit neurosciences at Imperial College London, UK. 